\title{
Stay smart: it's all about the materials for 4D printing in biomedicine
}

\section{Tristan Free ${ }^{*, 1}$}

${ }^{1}$ Future Science Ltd, Unitec House, 2 Albert Place, London, N3 1QB, UK; *Author for correspondence: t.free@future-science.com

BioTechniques 70: 191-193 (April 2021) 10.2144/btn-2021-0016

Where 3D printing was characterized by the new technologies and machinery, 4D printing is defined by the smart materials making waves in drug development and regenerative medicine.

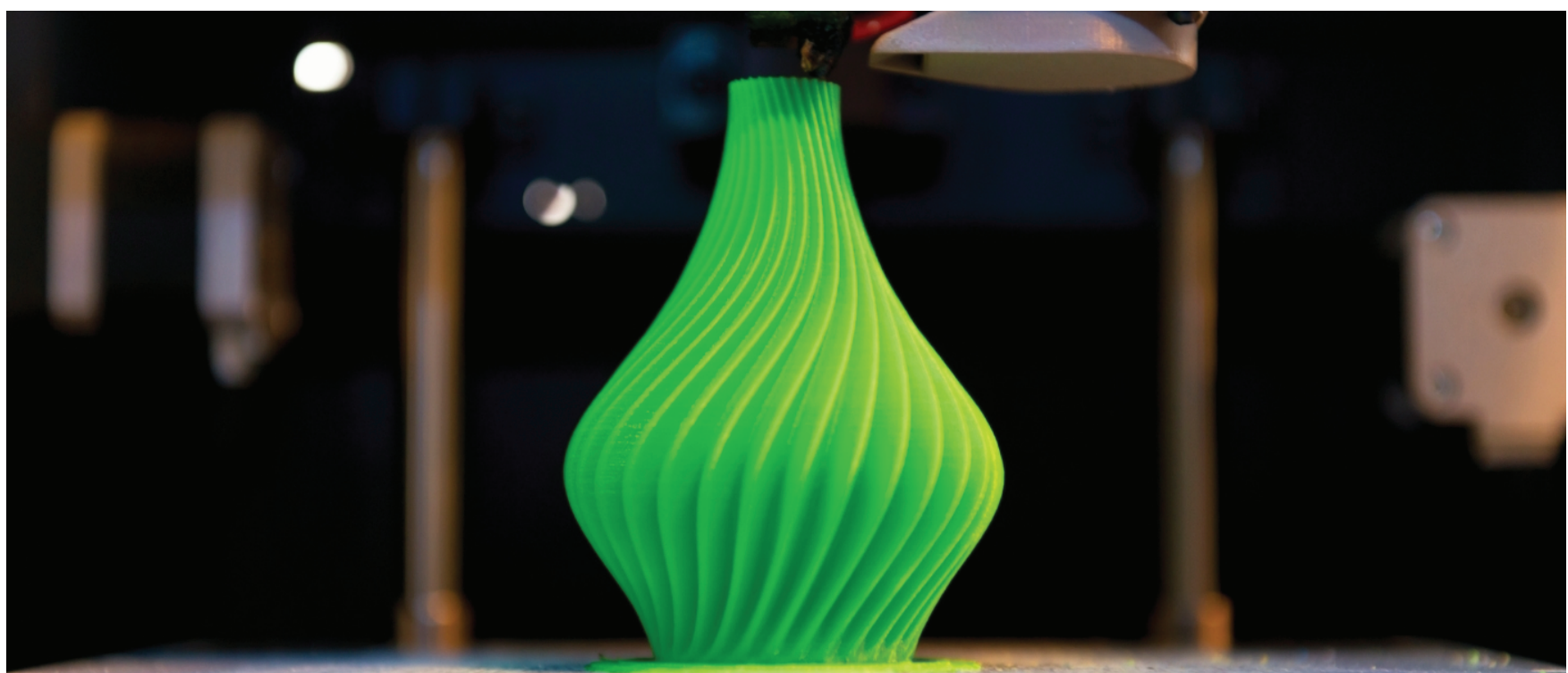

As 3D printing has developed over the last 30 years, there has been a litany of developments in the manufacturing industry. Expectations were high for these developments to carry through into the biomedical world and, in some respects, these expectations have been met. Significant benefits have been seen in surgical fields, enabling surgeons to construct models [1] and practice elements of a surgery prior to operation, manufacturing precise surgical instruments cheaper and creating tailored prosthetics.

When it comes to the more intricate practices of cell biology and drug development, however, the rigidity and permanence of the initial products of 3D printing have largely limited the technology's impact to the bioprinting of 3D cell structures used to model tissues and organs in vitro. To make the next definitive impact in the biomedical sciences, a new dimension needed to be incorporated into the products of $3 \mathrm{D}$ printing, allowing them to replicate the dynamic, changing environments that they intend to interact with: time.

This concept was coined '4D printing' during a TED talk [2] in 2013 by Skylar Tibbits (MIT, MA, USA). Still very much in their infancy, $4 \mathrm{D}$ printing technologies use 'smart' or 'dynamic' materials to manipulate the advances of 3D printing technologies to create products that can change shape over time or in response to various stimuli. Aided by advances in these smart materials, this improvement has dramatically expanded the potential of 3D printing in numerous fields of the life sciences.

\section{Advances in smart materials}

The nature of the stimuli that impact smart materials, along with their effects, vary dramatically depending on the specific molecules incorporated into the material. Broadly, these stimuli can be separated into three categories: physical, which include temperature, light, ultrasound, magnetic and electric fields; chemical, including $\mathrm{pH}$ and moisture; and biological stimuli, characterized by a response to biological molecules, such as an antibody, enzyme, metabolite or receptor [3].

To program each material to respond to these various stimuli requires an array of different molecules and techniques, such as the incorporation of chromophores or photochromatic dyes into a polymer matrix to manipulate a material to respond to light - switching polarity, conformation or changing its hydrophilicity. Conversely, to respond to a biological stimulus the polymer will need to be labeled with a receptor or ligand to allow it to interact with a desired molecule. This great collection of available alterations allows materials to be created that respond to several stimuli, known as multi-responsive materials [3]. 

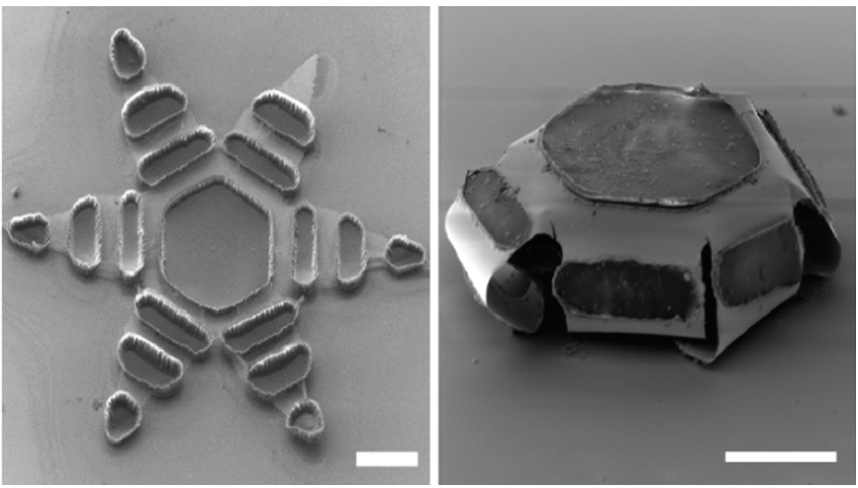

Figure 1. Open theragripper (left) and in closed, gripping conformation (right).

Reprinted from Johns Hopkins University [8].

One particularly useful result of this multi-responsive capability is the ability to design materials with shape memory [4]. These materials can be designed with a default shape that can be 'programmed' into an alternate temporary shape by the application of a specific stimulus and then 'recovered' by a second stimulus [5].

Through a combination of these methods, smart materials can be developed that divide into two different categories of shapememory materials, those that directly impact cells by releasing an active molecule in response to a signal and those that use their intelligent structure to facilitate and stimulate the growth of cells [5]. These two applications have clear implications for the fields of drug development and regenerative medicine respectively.

\section{Directing drug delivery}

A key example of an application for these smart materials in drug development is the ability to package the active ingredient of the drug into a 4D-printed delivery device. This device can then serve several purposes, containing the active ingredient until it encounters the target environment that the smart material has been modified to respond to or anchoring to a specific site and releasing the active ingredient at that location.

This application could be particularly useful in the development of drugs to treat gastrointestinal (GI) disorders. Often, the drugs administered to the GI tract are cleared by the mechanical action of the tract before the entirety of the dose can be absorbed by the body, reducing the impact of treatment.

\section{Gastroretentive drug delivery systems}

To address this requirement, 'theragrippers' were pioneered in 2014 by the Gracias and Selaru labs at The Johns Hopkins University (MD, USA), referring to devices composed of rigid poly(propylene fumarate) segments and stimuli-responsive hinges [6]. At temperatures above $32^{\circ} \mathrm{C}$ these devices close, latching onto the tissue that they first come in to contact with at the site of administration, allowing the therapeutics to be delivered at a constant, prolonged rate at a specific site.

In the latest development of theragrippers, the Gracias and Selaru labs took inspiration from the parasitic hookworm to design sixpointed star-shaped devices with sharp microtips (Figure 1). The team coated these devices in a thermosensitive paraffin wax that, when heated to body temperature, softens and allows the device to fold into its relaxed, closed state - like a spring that has been kept in its stretched conformation by the wax, returning to compression. The force of the device closing is sufficient to enable the microtips to penetrate the GI mucosa, rooting them in place. From this position, the active substance of choice is steadily released from a drug-eluting chitosan layer at the center of the structure [7].

The authors noted that the theragrippers can, "Reside within the Gl tract of live animals for 24 hours... We also observe a notable sixfold increase in the elimination half-life using theragripper-mediated delivery of a model analgesic ketorolac tromethamine."

An additional approach to enable slow, continued delivery of a therapeutic in the GI tract is to create a device that can be orally delivered into the stomach, where it then expands to a size large enough to block it from passing through the open pylorus. There it can release the drug slowly before degrading and being evacuated. There is, however, a risk that such a device could block the pylorus and lead to problems in the stomach [9].

To design a device that could fulfil the above requirements without blocking the pylorus an Italian research collaboration set out to use 4D printing to solve the problem. Using moisture-responsive poly(vinyl alcohol) (PVA) materials, the team used Fused Deposition Modeling 3D printing to create a structure with shape memory.

The team designed several different structures each with gaps wide enough to allow gastric contents to pass through it and out of the pylorus. Prototypes were printed using Fused Deposition Modeling or shaped from hot melt extruded rods and imbued with the drug allopurinol. Each of these prototypes was capable of being programmed into smaller sizes, able to fit into a swallowable capsule, which then recovered their original shape in response to immersion in $0.1 \mathrm{~N}$ hydrochloric solution at $37^{\circ} \mathrm{C}[9]$.

Though this approach did not provide an extended-release effect as dramatic as the theragrippers, this study provides a practical template for designing more effective iterations of these devices in the future. 
These drug delivery applications extend further than the GI tract, however. For instance, printing a device composed of either an acidic polymer, composed of several $-\mathrm{COOH}$ and $-\mathrm{SO}_{3} \mathrm{H}$ groups, or a basic polymer containing $-\mathrm{NH}_{2}$ groups, will allow the device to respond to $\mathrm{pH}$ [3]. These materials typically collapse or swell in response to a specific $\mathrm{pH}$, meaning drugs could be contained within the structure and targeted to be released in $\mathrm{pH}$-sensitive environments such as the mildly acidic tumor microenvironment [10]. From cancer to ulcerative colitis, $4 \mathrm{D}$ printing could be on the road to revolutionizing drug delivery.

\section{Revitalizing regenerative medicine}

One limitation of 4D-printed smart materials that has delayed the progress of the technique in regenerative medicine is their biocompatibility [11]. To function successfully in a clinical setting, biomaterials need to reflect the in vivo human tissues that they are intended to repair. What's more the stimuli required to induce dynamic change are often too damaging for in vivo settings.

Looking to improve the situation and rectify some of these shortcomings a team of researchers from Hallym University (Chuncheon, Republic of Korea) set out to design a cell-viable, biocompatible $4 \mathrm{D}$ bioprinting system to create biologically produced tissue mimetic scaffolds.

The team used a silk fibroin (SF) bioink, known as Sil-Ma, that they had previously developed. This sophisticated, natural protein-based bioink can be photopolymerized into a hydrogel. Utilizing Sil-Ma with digital light processing (DLP) 3D printing, which can increase cell viability by over $85 \%$ compared to other techniques [12], the team was able to create a hydrogel with differing bilayers. The resulting hydrogel was responsive to moisture - a naturally occurring and gentle stimulus - and highly biocompatible.

Using finite element analysis simulations, the team examined how alterations of the Sil-Ma bioink could influence changes in the hydrogel's shape. Integrating two different cell types into the hydrogel the team attempted to replicate a trachea-like structure, which was then implanted into the damaged trachea of a rabbit. After 8 weeks of observation epithelial and cartilage tissues were found to be growing at the intended sites [11].

A subsequent study from the University of Illinois (SA), released in February 2021, has utilized similar hydrogel technologies to develop biocompatible scaffolds that could drive the field towards successful vascular regeneration and bone reconstruction [12].

To create these hydrogels the team used oxidized and methacrylated alginate (OMA) and methacrylated gelatin (GelMA). The methacrylation of these products plays a key role in enabling their photocrosslinking and also contributes the 'Ma' aspect of their names - as with Sil-Ma. Combining these two polymers to form a bilayered hydrogel results in the hydrogel folding into a C-shape when in contact with water, due to the difference in water absorption between each layer [13].

The team found that by altering properties, such as polymer crosslink density, in each layer of the hydrogel, they could influence the rate at which the product changed shape and how dramatically the shape changed. Make the hydrogel bend enough and it can successfully form a full tube-type structure, providing the groundwork for the development of vascular models and scaffolds to help repair damaged vasculature [13].

What's more, these scaffolds were able to viably host the highest density of cells ever recorded on a 4D structure, even hosting bone marrow stem cells to deliver bone- and cartilage-like tissues. Commenting on the value of the cells, first author Yu Bin Lee noted that "This system holds promise for tissue engineering but may also be used to study the biological processes involved in early development."

The applications of $4 \mathrm{D}$ printing in the biomedical sciences are already numerous, creating models for the study of disease and development, improving therapeutic efficacy and healing damaged tissues. Yet, as smart materials get smarter, more biocompatible and respond to further stimuli with greater sensitivity and variation there is almost no predicting how many fields could be revolutionized by this new brand of technology. For now though, regenerative medicine and drug delivery lead the way in showcasing the benefits of embracing the fourth dimension.

\section{References}

1. BioTechniques.com. Craniopagus conjoined twins separated: the case of Safa and Marwa. www.biotechniques.com/whole-genome-studies/separating-craniopagus-conjoined-twins-th e-case-of-safa-and-marwa/

2. TED. The emergence of $4 \mathrm{D}$ printing. www.ted.com/talks/skylar_tibbits_the_emergence_of_4d_printing?language=en

3. Zhou W, Qiao Z, Zare E et al. 4D-printed dynamic materials in biomedical applications: chemistry, challenges, and their future perspectives in the clinical sector. J. Med. Chem. 63(15), 8003-8024 (2020).

4. Shie $M-Y$, Shen Y-F, Astuti A et al. Review of polymeric materials in $4 \mathrm{D}$ printing biomedical applications. Polymers. 11(11), 1864 (2019).

5. Shakibania S, Ghazanfari L, Raeeszadeh-Saemazdeh M, Khakbiz M. Medical application of biomimetic 4D printing. Drug Dev. Ind. Pharm. 1-31, doi:10.1080/03639045.2020.1862179 (2020) (Epub ahead of print).

6. Malachowski k, Breger J, Kwag H et al. Stimuli-responsive theragrippers for chemomechanical controlled release. Angew. Chem. Int. Ed. Engl. 53(31), 8045-8049 (2014).

7. Ghosh A, Li L, Xu L et al. Gastrointestinal-resident, shape-changing microdevices extend drug release in vivo. Sci. Adv. 6(44), eabb4133 (2020).

8. Johns Hopkins University. Johns Hopkins researchers take inspiration from parasitic work for medicine delivery. https://hub.jhu.edu/2020/11/25/theragripper-gi-tract-medicine-delivery/

9. Melocchi A, Uboldi M, Inerardi N et al. Expandable drug delivery system for gastric retention based on shape memory polymers: Development via 4D printing and extrusion. Int. J. Pharm. 571,118700 (2019).

10. Expansile nanoparticles: synthesis, characterization, and in vivo efficacy of an acid-responsive polymeric drug delivery system. J. Am. Chem. Soc. 131(7), 2469-2471 (2009).

11. Kim S, Seo Y, Yeon Y et al. 4D-bioprinted silk hydrogels for tissue engineering. Biomaterials 260, 120281 (2020).

12. Kim S, Yeon Y, Lee J et al. Precisely printable and biocompatible silk fibroin bioink for digital light processing 3D printing. Nat. Commun. 9, 1620 (2018).

13. Lee $\mathrm{Y}$, Jeon $\mathrm{O}$, Lee S, Ding A, Wells D, Alsberg E. Induction of four-dimensional spatiotemporal geometric transformations in high cell density tissues via shape-changing hydrogels. Adv. Funct. Mater. doi:10.1002/adfm.202010104 (2021) (Epub ahead of print). 
